\title{
General anesthetic and the risk of dementia in elderly patients: current insights
}

This article was published in the following Dove Press journal:

Clinical Interventions in Aging

24 September 2014

Number of times this article has been viewed

\author{
Maria Hussain' \\ Miles Berger ${ }^{2}$ \\ Roderic G Eckenhoff ${ }^{3}$ \\ Dallas P Seitz' \\ 'Division of Geriatric Psychiatry, \\ Department of Psychiatry, Queen's \\ University; ${ }^{2}$ Anesthesiology \\ Department, Duke University \\ Medical Center, Durham, NC, USA; \\ ${ }^{3}$ Department of Anesthesiology \\ and Critical Care, University \\ of Pennsylvania Perelman School \\ of Medicine, Philadelphia, PA, USA
}

Correspondence: Maria Hussain Kingston Geriatric Psychiatry Outreach Team, 640 Cataraqui Woods Drive, Unit 2, Kingston ON K7P 2Y5, Canada Email7mhI8@queensu.ca
Abstract: In this review, we aim to provide clinical insights into the relationship between surgery, general anesthesia (GA), and dementia, particularly Alzheimer's disease (AD). The pathogenesis of $\mathrm{AD}$ is complex, involving specific disease-linked proteins (amyloid-beta $[A \beta]$ and tau), inflammation, and neurotransmitter dysregulation. Many points in this complex pathogenesis can potentially be influenced by both surgery and anesthetics. It has been demonstrated in some in vitro, animal, and human studies that some anesthetics are associated with increased aggregation and oligomerization of $A \beta$ peptide and enhanced accumulation and hyperphosphorylation of tau protein. Two neurocognitive syndromes that have been studied in relation to surgery and anesthesia are postoperative delirium and postoperative cognitive dysfunction, both of which occur more commonly in older adults after surgery and anesthesia. Neither the route of anesthesia nor the type of anesthetic appears to be significantly associated with the development of postoperative delirium or postoperative cognitive dysfunction. A meta-analysis of case-control studies found no association between prior exposure to surgery utilizing GA and incident $\mathrm{AD}$ (pooled odds ratio $=1.05, P=0.43$ ). The few cohort studies on this topic have shown varying associations between surgery, GA, and AD, with one showing an increased risk, and another demonstrating a decreased risk. A recent randomized trial has shown that patients who received sevoflurane during spinal surgery were more likely to have progression of preexisting mild cognitive impairment compared to controls and to patients who received propofol or epidural anesthesia. Given the inconsistent evidence on the association between surgery, anesthetic type, and AD, well-designed and adequately powered studies with longer follow-up periods are required to establish a clear causal association between surgery, GA, and AD.

Keywords: surgery, anesthesia, Alzheimer's disease

\section{Background}

With advances in health care and increasing life expectancy, the population of older adults is increasing in most countries. It is estimated that the population aged 60 years and above will rise from 287 million in 2013 to 417 million in 2050 in the developed world, and from 554 million to 1.6 billion in developing countries. ${ }^{1}$ Advancing age is the strongest risk factor for the development of dementia, ${ }^{2}$ and dementia has been identified as a major current health care challenge. ${ }^{3}$ The worldwide prevalence of dementia in 2001 was estimated at 24.3 million, and will double every 20 years to an estimated prevalence of 42.3 million in 2020, and 81.1 million in 2040. ${ }^{4}$ Alzheimer's disease $(\mathrm{AD})$ is the most common form of dementia among older adults, accounting for about two-thirds of all cases..$^{5}$ The burden of dementia on society is substantial: in the United States, the estimated annual direct costs associated with AD in 2013 were $\$ 203$ billion. $^{6} \mathrm{AD}$ is also a leading cause for admission to long-term care facilities ${ }^{7}$ as well as a risk factor for hospitalization. ${ }^{8}$ Dementia care is particularly time intensive, 
and, in 2012, caregivers provided 17.5 billion hours of care to $\mathrm{AD}$ patients in the US. ${ }^{6} \mathrm{AD}$ is essentially irreversible, and approaches to prevention or treatment have not emerged.

$\mathrm{AD}$ is a multifactorial disease, and its risk factors can be categorized into non-modifiable and potentially modifiable ones. In addition to advancing age, other non-modifiable risk factors include female $\operatorname{sex}^{9,10}$ and genetic risk factors for both early-onset and late-onset dementia. ${ }^{11}$ Apolipoprotein E (ApoE) is associated with amyloid plaques and neurofibrillary tangles in $\mathrm{AD}$, and ApoE4 polymorphisms have been associated with sporadic AD. ${ }^{11,12}$ There are several potentially modifiable risk factors associated with AD. Vascular risk factors include diabetes mellitus, ${ }^{13}$ hypertension, and hypercholesterolemia. ${ }^{14}$ Possible nutritional risk factors include low levels of vitamin B12 and folate, as well as hyperhomocysteinemia. ${ }^{15}$ Head injuries are another potentially preventable risk factor for AD. ${ }^{16}$ Reducing these modifiable risk factors, prior to the onset of cognitive symptoms, could significantly lower AD prevalence. ${ }^{17}$ For example, lifestyle changes that could delay the onset of dementia by 2 years would translate into a reduction of $36 \%$ of dementia cases, and could also yield 30-year reduction in total economic burden of CAD219 billion in Canada alone. ${ }^{18}$

As the population ages, surgery is being performed more frequently, and in progressively older adults. ${ }^{19}$ In the US, approximately $35 \%$ of all surgical procedures are performed on adults older than 65 years. ${ }^{20}$ There has been increasing concern and research investigating whether cognitive sequelae such as postoperative delirium (POD) or postoperative cognitive dysfunction (POCD) are related to specific surgical and/or anesthetic factors. POD has been defined as an acute change in cognitive status characterized by fluctuating attention and consciousness, which typically occurs soon after surgery, ${ }^{21}$ while POCD is characterized by more persistent cognitive difficulties, including memory and attention problems, as well as executive dysfunction, and occurs commonly after surgery. ${ }^{22}$ Cognitive dysfunction is relatively common during the postoperative course of older adults, and anesthetics have been thought to possibly contribute to this. These cognitive changes can be both short and long term. Short-term cognitive dysfunction (days to months) encompasses both POD and POCD, while some patients may also experience longer-term cognitive changes (eg, greater than 6 months) postoperatively.

In this review, we will provide an overview of the relationship between surgery and anesthesia and subsequent cognitive changes including $\mathrm{AD}$ and related forms of dementia. We will first review the pathology of human AD, then we will discuss possible mechanisms by which surgery and anesthesia may produce cognitive changes or dementia, and then review biomarker evidence for these mechanisms. This review will also outline the epidemiological evidence for $\mathrm{AD}$ risk after exposure to surgery and general anesthesia (GA). We will identify gaps in the current literature and possible areas of future research.

\section{Pathophysiology of dementia in humans}

The diagnostic criteria for dementia published by the Alzheimer's Association define dementia as the development of cognitive or neuropsychiatric symptoms that are associated with a decline in the individual's previous level of functioning, encompassing multiple cognitive domains, and that are not better explained by delirium or another major psychiatric disorder. ${ }^{23}$ Most recently, guidelines have also incorporated biomarker evidence within these diagnostic criteria. These biomarker changes can include either decreased levels of amyloid-beta $(A \beta)$, together with increased total tau or phosphorylated tau in the cerebrospinal fluid (CSF) of the individual. ${ }^{24}$

The molecular neuropathology of AD reveals two major hallmarks: extracellular plaques consisting largely of $\mathrm{A} \beta$ aggregates, and intracellular neurofibrillary tangles composed of hyperphosphorylated tau protein. The amyloid cascade hypothesis states that some forms of the $\mathrm{A} \beta$ peptide are neurotoxic and cause abnormal phosphorylation of tau, leading to tau forming paired helical filaments which result in neurofibrillary tangles. This cascade also results in mitochondrial damage, calcium dysregulation, and, ultimately, apoptosis and neurodegeneration. ${ }^{25}$ It has been postulated that amyloid changes precede the onset of clinical symptoms of dementia by a decade or more. ${ }^{26,27}$ Results from 128 participants enrolled in the Dominantly Inherited Alzheimer Network show that changes reflecting AD pathology begin 25 years before expected symptom onset. ${ }^{28} \mathrm{~A} \beta$ peptide levels in CSF became abnormal first, and were followed by amyloid accumulation and cerebral atrophy and then impaired cerebral glucose metabolism.

This hypothesis has been strongly supported by genetic, biomarker, and autopsy studies. Autopsy studies have consistently found amyloidopathy and tauopathy in the brains of those diagnosed with AD. ${ }^{29,30}$ Further, biomarker studies show that $\mathrm{A} \beta$ peptide concentration in CSF is inversely related to the degree of $\mathrm{AD}$ pathology, ${ }^{31}$ likely because of sequestration in plaque or diminished synaptic activity. Interestingly, both amyloid plaque and reduced CSF A $\beta$ have been described in 
some older adults with no clinical symptoms indicative of a cognitive impairment. ${ }^{32}$ This suggests that amyloidopathy alone is insufficient for the development of clinical symptoms. The presence of tau in CSF, although not specific for $\mathrm{AD}$, indicates neuronal injury. Tau is usually elevated in the CSF of individuals with $\mathrm{AD}^{33}$ and other tauopathies such as frontotemporal dementia. ${ }^{34}$ The ratio of total tau to $A \beta$ is currently used as a diagnostic adjunct for AD. ${ }^{35}$

Positron emission tomography (PET) scans have been used to quantify the extent of amyloid deposition in vivo. For example, compounds that preferentially bind to amyloid fibrils (11-C Pittsburgh compound B, ${ }^{18} \mathrm{~F}$-florbetapir, ${ }^{18} \mathrm{~F}$ flutemetamol, and ${ }^{18} \mathrm{~F}$-florbetaben) are available, and studies to date indicate they sensitively report cerebral amyloidosis on PET scans. ${ }^{36,37}$ Since amyloidopathy reflects an early stage in the pathology, PET scanning may allow risk stratification well before symptoms begin. Further, PET scans demonstrate a decreased uptake of ${ }^{18} \mathrm{~F}$-fluorodeoxyglucose, a marker of cerebral glucose metabolism, in patients with $\mathrm{AD}$, likely reflecting the decreased neuronal mass secondary to neurodegeneration. ${ }^{27}$ The early detection of risk is important because it may allow patients to modify AD risk factors. However, it is now clear that false positives occur with these and other biomarkers, which has potential implications when considering the use of these biomarkers.

Multiple neurotransmitters have also been studied in the context of $\mathrm{AD}$. Cholinergic dysfunction has been implicated in the clinical symptoms of dementia, ${ }^{38-40}$ and postmortem studies have identified a significant deficiency in the activity of choline acetyltransferase in the brains of individuals with dementia. ${ }^{41} \mathrm{AD}$ is also associated with a loss of cholinergic neurons, and acetylcholine modulates higher functions of the brain such as memory, learning, and attention. The cholinergic deficit seen in AD is associated with the degree of cognitive dysfunction, providing the rationale for the use of acetylcholinesterase inhibitors in AD. Other neurotransmitters, including excitatory amino acids (eg, glutamate), can be neurotoxic, and may play a role in the pathophysiology of $\mathrm{AD} .{ }^{42,43}$ Changes in neurotransmitter function may also provide potential links between exposure to GA, surgery, and cognitive changes following surgery.

\section{Basic science studies of AD pathophysiology associated with surgery and anesthetic agents}

Several mechanisms linking anesthetics to worsening of AD pathology from basic science studies have been proposed, providing a potential causal connection between anesthesia exposure and development of AD. ${ }^{44}$ An in vitro study showed that the volatile anesthetics halothane and isoflurane increase oligomerization and cytotoxicity of amyloid peptides. ${ }^{45}$ Both in vitro and animal studies show that sevoflurane and isoflurane can cause neuronal apoptosis by activating caspase and $\mathrm{A} \beta$ protein aggregation. ${ }^{46,47}$ Further, after exposure to isoflurane, mice displayed behavioral impairments and increased mortality. ${ }^{48}$ Propofol, another intravenous anesthetic, also increases tau phosphorylation in the mouse hippocampus. ${ }^{49}$ Overall, information has shown potential linkages between surgery and changes in AD-related pathology, as well as between anesthesia and changes in AD-related pathology.

GA could theoretically also contribute to cognitive deficits by altering central cholinergic transmission through nicotinic and muscarinic receptors. A decrease in acetylcholine neurotransmission facilitates some of the desired effects of GA, including analgesia, amnesia, immobility, and hypnosis. ${ }^{50}$ Some anesthetics inhibit N-methyl-D-aspartate (NMDA) receptors, leading to decreased glutamate release and a subsequent decrease in excitatory activity. ${ }^{51}$ However, prolonged exposure to NMDA antagonists, such as ketamine, may upregulate NMDA receptors following sustained blockade, which, on removal of drug, leads to excitotoxicity and apoptosis via an increase in calcium influx. ${ }^{52}$

There is a large body of literature from animal studies examining the effects of surgery on cognitive function. Cognitive decline in aged mice following surgery has been associated with microgliosis, A $\beta$ production, and tau protein hyperphosphorylation in the hippocampus. ${ }^{53}$ The role of neuroinflammation as a result of surgery has also been extensively studied. In mice, surgery leads to the production of tumor necrosis factor $\alpha$ (TNF- $\alpha$ ), which subsequently disrupts the blood-brain barrier, causing an infiltration of inflammatory macrophages in the brain parenchyma, specifically the hippocampus. ${ }^{53}$ The levels of the inflammatory cytokines IL-1 $\beta$ and IL-6 have also been shown to increase in mice that underwent surgery, compared to mice who only received anesthesia. ${ }^{54}$ In this same study, the levels of cytokines in the group that received anesthesia without surgery were comparable to anesthesia-naïve control mice. ${ }^{54}$ This work demonstrates that the inflammatory response and resultant cognitive deficits may largely be a result of surgery rather than due to any specific effects of GA. However, these findings only reflect data from animal studies and it is unclear to what extent these findings extend to humans. Thus, in the future, data from studies investigating the effects of specific anesthetics on neurotransmitter function may provide some 
guidance for the selection of anesthetics to reduce cognitive dysfunction in the elderly.

\section{AD biomarkers associated with exposure to surgery and anesthesia in humans}

Longitudinal studies with a prolonged follow-up period would be required to causally link surgery and anesthesia with the development of clinically diagnosed AD. In the absence of these studies, it may be possible to examine the effects of GA on more proximal AD CSF or neuroimaging biomarkers. Studies have shown that AD biomarkers are altered in the CSF of individuals undergoing cardiac and noncardiac surgery. ${ }^{55,56}$ Palotás et al conducted a small study on the CSF of 14 patients without dementia undergoing coronary artery bypass graft surgery (CABG). CSF samples were collected prior to surgery and 1 week and 6 months postoperatively. Levels of $A \beta$, tau, and S100beta (a marker of nonspecific brain injury) were measured. All three CSF markers increased significantly 1 week postoperatively. At 6 months, the increase in S100beta subsided, and $A \beta$ peptide decreased to below baseline, while tau continued to rise. ${ }^{55}$ The 6-month values in CSF biomarkers were roughly comparable to those generally observed in patients with $\mathrm{AD}$, although this particular study did not have an AD control group, and the data for AD CSF biomarker levels were cited from other studies. Another limitation of this study is the small sample size. Further, although the decline in cognitive function was statistically significant, it is unclear to what extent the decline in cognitive function correlated with biomarker changes among individual patients. Shiiya et al reported the findings of 28 patients who underwent prosthetic replacement of the descending thoracic or thoracoabdominal aorta. ${ }^{57} \mathrm{CSF}$ levels of tau and S100beta were measured immediately before and up to 72 hours after surgery. The levels of CSF tau and S100beta were only increased in individuals with brain infarction or transient neurologic dysfunction; no increase in these CSF biomarkers was found in individuals who did not have neurologic complications. Tang et al studied the CSF of eleven patients undergoing idiopathic CSF leak correction for biomarkers of AD. All patients received GA, with either intravenous propofol and remifentanil, or the inhalational anesthetic sevoflurane. CSF samples were collected before surgery and up to 48 hours postoperatively. Levels of $A \beta$ peptide, total tau and phosphorylated tau, S100beta, interleukins 6 and 10, and TNF- $\alpha$ were measured. Mean $A \beta$ peptide concentrations were statistically unchanged, whereas levels of total tau were significantly increased at 6 and 24 hours postoperatively. Phosphorylated tau was also increased, although not to the same extent as total tau. S100beta, and the interleukins 6 and 10 and TNF- $\alpha$, were increased in the CSF of these individuals as well. ${ }^{56}$ The aforementioned studies indicate biomarker changes that may reflect an increase in AD risk after anesthesia and surgery. Another study reported the findings of changes in the CSF of ten patients who underwent cardiac valve replacement surgery with cardiopulmonary bypass. CSF samples were collected a day before surgery and 24 hours postoperatively, and assessed for levels of total tau, neurofilament light chain protein, neuron-specific enolase (indicator of neuronal damage), S100beta, glial fibrillary acidic protein (marker of glial cell injury), and the cytokines interleukins 6 and $8 .{ }^{58}$ Levels of S100beta and glial fibrillary acidic protein increased by $35 \%$ postoperatively, while levels of total tau, neurofilament light chain protein, and neuron-specific enolase did not change significantly. The levels of both interleukin 6 and 8 in the CSF increased significantly after anesthesia and surgery as well.

The above studies demonstrate that surgery and anesthesia may be associated with markers of neuronal injury, although their results should be interpreted with caution due to the small size and the unclear correlation between biomarker changes and cognitive deficits.

\section{Cognitive changes associated with surgery and anesthesia in human populations}

Surgery and anesthesia may be associated with neurocognitive symptoms in the elderly. The commonly seen syndromes are POD and POCD. These early postoperative cognitive changes could possibly be linked to development of persistent cognitive deficits consistent with AD.

POD occurs commonly after surgery in older adults, and the average incidence of POD has been estimated at $36.8 \%,{ }^{21}$ although rates as high as $73.5 \%$ have also been reported. ${ }^{59}$ Additionally, the depth of anesthesia as measured by bispectral index (BIS) has also been investigated in relation to POD, and there is evidence suggesting that depth of anesthesia may be related to the risk of delirium. ${ }^{60}$ Radtke et al showed that incidence of POD in surgical patients whose depth of anesthesia was monitored by BIS was $16.7 \%$, compared to $21.4 \%$ in the control group $(P=0.036) .{ }^{61}$ Another study showed similar results, with the rate of POD being significantly lower in the BIS group than in the control group (15.6\% versus $24.1 \%, P=0.01){ }^{62}$

However, results of a recent meta-analysis show that GA does not appear to confer higher risk to the development of POD as compared to regional anesthesia (RA). ${ }^{63}$ Further, 
it has also been demonstrated that individuals who have baseline cognitive impairment are not at increased risk of developing POD if they receive GA versus regional anesthesia. ${ }^{64}$ It is evident from these studies that older adults are vulnerable to developing POD and that there are several factors, including premorbid cognitive status, which put them at risk. Long-term consequences of POD can be substantial, and it has been reported that POD is associated with prolonged cognitive impairment in patients who have cardiac surgery. ${ }^{65}$ Additionally, POD was associated with an increased risk of developing dementia among older adults without dementia undergoing hip fracture surgery at 5-year follow-up. ${ }^{66}$ Another study showed that patients who developed delirium after hip fracture (pre- and postoperatively) had a higher rate of incident dementia at 6-month follow-up (adjusted odds ratio $=10.5){ }^{67}$

Long-term consequences of POCD are significant, as patients with POCD have higher mortality rates at 3 months and 1 year compared to controls. ${ }^{68,69}$ The reported incidence of POCD has varied considerably across studies, partly due to changes in how it was measured: there is no uniform consensus definition of what degree of cognitive impairment qualifies as POCD. Studies on POCD have been criticized due to either the presence of a poorly matched control group or the complete absence of one. ${ }^{70}$ In a large prospective study, cognitive decline at discharge was reported in $53 \%$ of the patients who underwent coronary artery bypass surgery. ${ }^{71}$ The incidence decreased to $36 \%$ at 6 weeks and $24 \%$ at 6 months. However, there was no control group in this study. In a systematic review, the pooled prevalence of POCD was estimated to be $22.6 \%$ in the 2 months after surgery. ${ }^{72}$ A large cohort study reported the rates of POCD in patients undergoing major noncardiac surgery: the prevalence of POCD was $25.8 \%$ at 1 week and $9.9 \%$ at 3 months postoperatively in the surgical patients, whereas the prevalence of cognitive dysfunction in the nonsurgical control group was $3.4 \%$ and $2.8 \%$ at 1 week and 3 months, respectively $(P<0.0001$ and $P=0.0037) .{ }^{22}$ Several studies have reported risk factors associated with the development of POCD. The etiology of POCD likely includes surgical, anesthetic, and patient-related factors. In one study, independent risk factors for POCD 3 months postoperatively were advancing age, history of cerebrovascular accident, lower education level, and POCD at discharge. ${ }^{68}$ The role of anesthesia in the development of POCD has also been investigated. A meta-analysis by Mason et al investigated whether the type of anesthesia affected the development of POCD. ${ }^{63}$ In contrast to the studies of POD mentioned earlier in this review, GA was associated with an odds ratio of 1.34 for the development of POCD when compared to regional anesthesia. A double-blind, randomized controlled trial of 80 patients aged 65-75 years conducted by Rörtgen et al investigated whether the choice of inhalational anesthetic (desflurane versus sevoflurane) affected the rate of development of POCD in patients undergoing elective surgery. ${ }^{73}$ This study did not find any significant difference in the incidence of POCD between the two groups.

Studies have investigated whether an association exists between the depth of anesthesia and development of POCD. Ballard et al conducted a 52-week prospective cohort study with a nested randomized controlled trial wherein they studied the effects of optimization of anesthesia and cerebral oxygen saturation. ${ }^{74}$ The patient population included 192 adults $>60$ years old undergoing elective orthopedic or abdominal surgery and 138 matched controls. At 52 weeks, mild and moderate POCD were significantly lower in the intervention group compared to the nonintervention surgical group ( $84 \%$ versus $56 \%, P=0.015)$. Severe POCD was also lower in the intervention group $(3.7 \%$ versus $12.5 \%$, $P=0.36$ ). One of the tests used to measure cognitive function was the Mini-Mental ${ }^{\circledR}$ State Examination (MMSE), which may not be sensitive to milder cognitive changes and is also prone to potential learning effects. ${ }^{75}$ Therefore, these results should be interpreted with caution. Radtke et al showed that there was no statistically significant difference in the rate of POCD in patients whose depth of anesthesia was monitored by BIS versus the control group utilizing routine anesthetic monitoring. ${ }^{61}$ However, another group of investigators showed that, although there was no statistically significant difference in the rate of POCD at 7 days between patients who had BIS monitoring and those in the control group, rates of POCD in the intervention group were lower at 3 months compared to the control group (10.2\% versus $14.7 \%$, adjusted odds ratio $=0.67, P=0.025) .{ }^{62}$

There are methodological limitations in the existing studies of POCD related to the measures used to define POCD. These limitations include: potential learning effects; floor and ceiling effects; test sensitivity; timing of postoperative testing; and lack of diagnostic criteria for POCD. ${ }^{76}$ Therefore, it is essential that diagnostic criteria for POCD be standardized, as well as recommendations be made for the use of standardized neuropsychological tests.

There are certain similarities between POCD and AD, including advanced age being the strongest risk factor for both. Additionally, the clinical presentations described in the literature for POCD can encompass dysfunction in multiple 
cognitive domains similar to $\mathrm{AD}$, for example, memory impairment, attention and concentration difficulties, and executive dysfunction. However, the association between POCD and development of dementia is unclear at this time. The International Study of Postoperative Cognitive Dysfunction (ISPOCD) group recently published a cohort follow-up study, presenting the results of 686 patients from the original ISPOCD1 and ISPOCD2 studies ${ }^{22,77-80}$ who had been followed for a median of 11.1 years. The outcome was the first occurrence of dementia in patients who had been diagnosed with POCD at 1 week and 3 months after surgery. The hazard ratio for the development of dementia was 1.16 for patients who had POCD at 1 week, and 1.50 for patients with POCD at 3 weeks. Even though this increase may appear to be clinically significant, due to the limited power of this study, the authors concluded that there was no statistically significant association between incident dementia and POCD at 1 week ( $P=0.74)$ and 3 months $(P=0.47)$ as compared to patients who did not have POCD. ${ }^{81}$ Further well-designed and adequately powered studies with a long follow-up period are needed to establish whether there is an association between POCD and dementia.

\section{Retrospective observational studies on the association between exposure to surgery or anesthesia and risk of dementia}

It is challenging to separately examine the independent effects of surgery and anesthesia on postoperative cognition and development of dementia. Surgery leads to a larger stress response in older adults and in those who have preexisting $\mathrm{AD}$ compared to younger patients and those without AD ${ }^{82}$ Surgery could influence cognition by altering cerebral perfusion and autoregulation. Lower cerebral perfusion pressure can lead to ischemia, and higher pressures can cause cerebral edema. ${ }^{83}$ Observational studies have demonstrated that certain types of surgeries, such as cardiovascular, genitourinary, abdominal, musculoskeletal, dermatological, and eye surgeries, may confer a higher risk of developing dementia, ${ }^{84,85}$ although it is unclear whether these specific surgery types are associated with greater alterations in cerebral perfusion and/or autoregulation, or whether the indication for surgery itself represents the direct risk factor.

Researchers have conducted observational studies in order to examine the relationship between prior exposure to GA and subsequent risk of dementia. Case-control studies investigating potential risk factors associated with dementia, and specifically $\mathrm{AD}$, have also assessed the risk of having anesthesia and surgery. This study design provides a matched control group in order to provide a more robust method of establishing an association. We published a meta-analysis of case-control studies examining the association between GA and AD. ${ }^{86}$ This review defined exposure to GA as any history of surgery under GA as compared to no history of surgery under GA (ie, the control group could either be nonsurgical or a surgical control group receiving regional anesthesia). The outcome was defined as a diagnosis of AD of any severity. Fifteen studies were included in this metaanalysis, including a total of 1,752 cases and 5,261 controls. Overall, there was no statistically significant association of $\mathrm{GA}$ with the development of $\mathrm{AD}$ (pooled odds ratio $=1.05$, $P=0.43$ ). Subgroup analyses compared GA exposure to regional anesthesia and the effect of cumulative anesthesia/ surgery exposures over the patient's lifetime. We found no difference in the odds ratio for developing AD after receiving GA compared to RA. There was also no statistically significant association between cumulative exposure to GA and development of AD.

Since the publication of that meta-analysis, additional studies have been published that have investigated the role of GA in the development of AD. Sprung et al conducted a retrospective population-based nested case-control study using the Rochester Epidemiology Project and the Mayo Clinic Alzheimer's Disease Patient Registry. ${ }^{87}$ They identified 877 cases of incident dementia recorded between January 1, 1985 and December 31, 1994, and sex- and aged-matched individuals who did not have a diagnosis of dementia in the incident year made up the control group. Exposure to anesthesia in the study group was determined by reviewing medical records for each patient after age 45 years and before the index year. Among cases, $70 \%$ of individuals had been exposed to GA compared to $72.5 \%$ of the control group. The authors did not find a significant association between exposure to anesthesia after age 45 years and the diagnosis of dementia $(P=0.27)$, and also found no association between the number of procedures or cumulative exposure to anesthesia and development of dementia $(P=0.51)$. A second case-control study with a large sample size was recently conducted in Taiwan using the Longitudinal Health Insurance Database. This study identified 5,345 patients from the insurance claims database who were over the age of 50 years and had a new diagnosis of dementia, and 21,380 patients without dementia. These groups were matched for age, sex, and index year. ${ }^{88}$ The authors divided GA into three categories: endotracheal tube intubation GA 
(ETGA), intravenous injection GA (IVGA), or intramuscular injection GA (IMGA), versus sedation only. The dementia group had greater exposure to ETGA, IVGA, and IMGA when compared to controls, whereas the rates of use of heavy sedation did not differ between the groups. Patients exposed to ETGA (odds ratio =1.34) or IVGA/IMGA (odds ratio $=1.28$ ) were at significantly higher risk of developing dementia in a dose-response relationship compared to the control group $(P<0.0001)$.

In addition to case-control studies, observational data are also available from cohort studies. Lee et al performed a retrospective cohort analysis to study the relationship between surgery, GA, and AD. The study population included individuals aged 55 years old or older without a diagnosis of $\mathrm{AD}$ prior to surgery. One group received GA and underwent regular $\mathrm{CABG}$, while the other group received only sedation for percutaneous transluminal coronary angioplasty for stent placement. In the 5-year follow-up period, patients who had undergone $\mathrm{CABG}$ and GA had a 1.7-fold increased risk of developing $\mathrm{AD}$ compared to those who had had sedation and percutaneous transluminal coronary angioplasty. ${ }^{85}$ Vanderweyde et al conducted a retrospective cohort study on two groups of surgical patients undergoing either prostate or hernia surgery under either GA or regional anesthesia. Their results showed that the patients who had received GA developed dementia less frequently than those who had received regional anaesthesia ${ }^{89}$ (adjusted hazard ratio $=0.65$ for GA), although the depth or amount of sedation in the regional anesthesia group was not measured. A retrospective cohort study was also performed in Taiwan using the Longitudinal Health Insurance Database. ${ }^{84}$ The study cohort comprised 24,901 patients 50 years and older who, since 1995, were anesthetized for the first time between January 1, 2004 and December 31, 2007. The control group had 110,972 patients who had not been exposed to anesthesia. The investigators recorded the first occurrence of a diagnosis of dementia (presenile dementia, senile dementia, or AD). In order to distinguish dementia from POCD, the diagnosis had to be recorded twice, with first occurrence being at least 3 months after administration of anesthesia to minimize the possibility of misdiagnosing POCD as dementia. In a 3- to 7-year follow-up period, the risk of dementia in the anesthesia group was significantly higher than in the control group (hazard ratio $=1.99, P<0.001)$. Within the anesthesia group, patients who received regional anesthesia were at a higher risk of developing dementia as compared to the GA group $(P<0.001)$. The authors concluded that patients undergoing anesthesia and surgery are at higher risk of developing dementia.

\section{Prospective clinical studies on the association between exposure to surgery or anesthesia and risk of dementia}

There are very few prospective clinical studies investigating the association between GA and AD. Recently, Liu et al conducted a prospective randomized parallel-group study on the effects of anesthesia on the progression of amnestic mild cognitive impairment (aMCI) to $\mathrm{AD}$ in a Chinese population..$^{90}$ The study group consisted of 180 patients who had a diagnosis of aMCI, a subtype of mild cognitive impairment in which memory problems are the predominant symptom. These patients were randomly assigned to receive sevoflurane, propofol, or lidocaine epidural anesthesia for lumbar spinal surgery. Sixty outpatients with the same cognitive diagnosis served as the control group. Neuropsychological tests were conducted at baseline before surgery, and CSF samples were obtained and levels of $A \beta$ peptide, total tau, and phosphorylated tau were measured. At 2-year follow-up, the number of patients who had progressed to AD did not differ between the two groups. Patients assigned to receive lidocaine epidural anesthesia or intravenous propofol anesthesia had the same rate of progression of aMCI as the control group, whereas the rate of progression to dementia was faster in the sevoflurane group $(P=0.005)$, and the number of progressive aMCI cases was higher compared to the other groups $(P=0.01)$. The authors concluded that sevoflurane anesthesia for lumbar spine surgery accelerated cognitive decline, but that further studies with a larger sample size and longer follow-up period were required to investigate this relationship further.

\section{Conclusion}

The prevalence of $\mathrm{AD}$ is predicted to rise with the aging population. Older adults are requiring the use of surgery and GA more frequently, and it is pertinent to examine whether surgery and GA are associated with the development of AD. In vitro and animal studies suggest that surgery and GA can accelerate AD pathology. Cognitive changes, including POD and POCD, after surgery and anesthesia are common in the elderly. However, it is unclear whether surgery and anesthesia increase the risk of longer-term cognitive disorders such as dementia and AD. Data from observational studies in humans on the relationship between surgery, GA, and AD have been inconsistent. A previously published metaanalysis failed to show an association between surgery, GA, and $\mathrm{AD}$ in case-control studies. Some studies published more recently do suggest a possible association between exposure 
to surgery and anesthesia and the development of AD. Given the gaps identified in the current research literature, there are several factors that should be considered in designing future studies in this important area. Future study designs should include a sufficient sample size; presence of a nonsurgical, non-anesthetized control group; assessment of preoperative cognition, using standardized neuropsychological testing for cases and controls; and possibly the use of biomarkers and neuroimaging for determining amyloid load and AD pathology. In summary, adequately powered prospective trials meeting the aforementioned criteria, and with a longer follow-up period, are required to determine whether exposure to surgery and/or GA are causally associated with the development of AD.

\section{Disclosure}

Dr Seitz has received honoraria from Eli-Lilly for participation in an advisory board. The authors report no other conflicts of interest in this work.

\section{References}

1. World Population Prospects: The 2012 Revision. Key Findings and Advance Tables. Working Paper No. ESA/P/WP.227. New York: United Nations, Department of Economic and Social Affairs, Population Division; 2013.

2. Lindsay J, Laurin D, Verreault R, et al. Risk factors for Alzheimer's disease: a prospective analysis from the Canadian Study of Health and Aging. Am J Epidemiol. 2002;156(5):445-453.

3. Berr C, Wancata J, Ritchie K. Prevalence of dementia in the elderly in Europe. Eur Neuropsychopharmacol. 2005;15(4):463-471.

4. Ferri CP, Prince M, Brayne C, et al; Alzheimer's Disease International. Global prevalence of dementia: a Delphi consensus study. Lancet. 2006;366(9503):2112-2117.

5. [No authors listed]. Canadian study of health and aging: study methods and prevalence of dementia. CMAJ. 1994;150(6):899-913.

6. Thies W, Bleiler L; Alzheimer's Association. 2013 Alzheimer's disease facts and figures. Alzheimers Dement. 2013;9(2):208-245.

7. Zimmerman S, Sloane PD, Williams CS, et al. Dementia care and quality of life in assisted living and nursing homes. Gerontologist. 2005; 45 Spec No 1(1):133-146.

8. Albert SM, Costa R, Merchant C, Small S, Jenders RA, Stern Y. Hospitalization and Alzheimer's disease: results from a community-based study. J Gerontol A Biol Sci Med Sci. 1999;54(5):M267-M271.

9. Lobo A, Launer L, Fratiglioni L, et al. Prevalence of dementia and major subtypes in Europe: a collaborative study of population-based cohorts. Neurologic Diseases in the Elderly Research Group. Neurology. 2000; 54(11 Suppl 5):S4-S9.

10. Miech RA, Breitner JC, Zandi PP, Khachaturian AS, Anthony JC, Mayer L. Incidence of AD may decline in the early 90 s for men, later for women: The Cache County study. Neurology. 2002;58(2): 209-218.

11. Corder E, Saunders A, Strittmatter W, et al. Gene dose of apolipoprotein E type 4 allele and the risk of Alzheimer's disease in late onset families. Science. 1993;261(5123):921-923.

12. Poirier J, Bertrand P, Poirier J, et al. Apolipoprotein E polymorphism and Alzheimer's disease. Lancet. 1993;342(8873):697-699.

13. Cheng G, Huang C, Deng H, Wang H. Diabetes as a risk factor for dementia and mild cognitive impairment: a meta-analysis of longitudinal studies. Internal Med J. 2012;42(5):484-491.
14. Craft S. The role of metabolic disorders in Alzheimer disease and vascular dementia: two roads converged. Arch Neurol. 2009;66(3):300-305.

15. Seshadri S, Beiser A, Selhub J, et al. Plasma homocysteine as a risk factor for dementia and Alzheimer's disease. N Engl J Med. 2002;346(7): 476-483.

16. Mortimer JA, French LR, Hutton JT, Schuman LM. Head injury as a risk factor for Alzheimer's disease. Neurology. 1985;35(2):264-267.

17. Barnes DE, Yaffe K. The projected effect of risk factor reduction on Alzheimer's disease prevalence. Lancet Neurol. 2011;10(9):819-828.

18. Rising Tide: The Impact of Dementia on Canadian Society. Toronto, ON: Alzheimer Society of Canada; 2010. Available from: http://www. alzheimer.ca/en/Get-involved/Raise-your-voice/Rising-Tide. Access date: August 29, 2014.

19. Etzioni DA, Liu JH, Maggard MA, Ko CY. The aging population and its impact on the surgery workforce. Ann Surg. 2003;238(2):170-177.

20. Bose R, Culley DJ, Groban L, et al. A Geriatric Anesthesiology Curriculum. 2007. Available from: http://www.sagahq.org/images/GeriCurric. pdf. Accessed August 29, 2014.

21. Dyer CB, Ashton CM, Teasdale TA. Postoperative delirium: a review of 80 primary data-collection studies. Arch Intern Med. 1995;155(5): 461-465.

22. Moller JT, Cluitmans P, Rasmussen LS, et al. Long-term postoperative cognitive dysfunction in the elderly ISPOCD1 study. ISPOCD investigators. International Study of Post-Operative Cognitive Dysfunction. Lancet. 1998;351(9106):857-861.

23. McKhann GM, Knopman DS, Chertkow H, et al. The diagnosis of dementia due to Alzheimer's disease: recommendations from the National Institute on Aging-Alzheimer's Association workgroups on diagnostic guidelines for Alzheimer's disease. Alzheimers Dement. 2011;7(3):263-269.

24. Dubois B, Feldman HH, Jacova C, et al. Advancing research diagnostic criteria for Alzheimer's disease: the IWG-2 criteria. Lancet Neurol. 2014;13(6):614-629.

25. Hardy JA, Higgins GA. Alzheimer's disease: the amyloid cascade hypothesis. Science. 1992;256(5054):184-185.

26. Jack CR Jr, Knopman DS, Jagust WJ, et al. Hypothetical model of dynamic biomarkers of the Alzheimer's pathological cascade. Lancet Neurol. 2010;9(1):119-128.

27. Sperling RA, Aisen PS, Beckett LA, et al. Toward defining the preclinical stages of Alzheimer's disease: recommendations from the National Institute on Aging-Alzheimer's Association workgroups on diagnostic guidelines for Alzheimer's disease. Alzheimers Dement. 2011;7(3):280-292.

28. Bateman RJ, Xiong C, Benzinger TL, et al. Clinical and biomarker changes in dominantly inherited Alzheimer's disease. $N$ Engl J Med. 2012;367(9):795-804.

29. Knopman DS, Parisi JE, Salviati A, et al. Neuropathology of cognitively normal elderly. J Neuropathol Exp Neurol. 2003;62(11):1087-1095.

30. Savva GM, Wharton SB, Ince PG, Forster G, Matthews FE, Brayne C. Age, neuropathology, and dementia. N Engl J Med. 2009;360(22): 2302-2309.

31. Fagan AM, Mintun MA, Mach RH, et al. Inverse relation between in vivo amyloid imaging load and cerebrospinal fluid Abeta42 in humans. Ann Neurol. 2006;59(3):512-519.

32. Aizenstein HJ, Nebes RD, Saxton JA, et al. Frequent amyloid deposition without significant cognitive impairment among the elderly. Arch Neurol. 2008;65(11):1509-1517.

33. Clark CM, Xie S, Chittams J, et al. Cerebrospinal fluid tau and betaamyloid: how well do these biomarkers reflect autopsy-confirmed dementia diagnoses? Arch Neurol. 2003;60(12):1696-1702.

34. Green AJ, Harvey RJ, Thompson EJ, Rossor MN. Increased tau in the cerebrospinal fluid of patients with frontotemporal dementia and Alzheimer's disease. Neurosci Lett. 1999;259(2):133-135.

35. Maddalena A, Papassotiropoulos A, Müller-Tillmanns B, et al. Biochemical diagnosis of Alzheimer disease by measuring the cerebrospinal fluid ratio of phosphorylated tau protein to beta-amyloid peptide 42 . Arch Neurol. 2003;60(9):1202-1206. 
36. Ikonomovic MD, Klunk WE, Abrahamson EE, et al. Post-mortem correlates of in vivo PiB-PET amyloid imaging in a typical case of Alzheimer's disease. Brain. 2008;131(6):1630-1645.

37. Newberg AB, Arnold SE, Wintering N, Rovner BW, Alavi A. Initial clinical comparison of ${ }^{18} \mathrm{~F}$-florbetapir and ${ }^{18} \mathrm{~F}$-FDG PET in patients with Alzheimer disease and controls. J Nucl Med. 2012;53(6):902-907.

38. Bartus RT, Dean RL, Beer B, Lippa AS. The cholinergic hypothesis of geriatric memory dysfunction. Science. 1982;217(4558):408-414

39. Fuld PA. Test profile of cholinergic dysfunction and of Alzheimer-type dementia. J Clin Neuropsychol. 1984;6(4):380-392.

40. Sims NR, Bowen DM, Allen SJ, et al. Presynaptic cholinergic dysfunction in patients with dementia. J Neurochem. 1983;40(2):503-509.

41. Perry EK, Gibson PH, Blessed G, Perry RH, Tomlinson BE. Neurotransmitter enzyme abnormalities in senile dementia. Choline acetyltransferase and glutamic acid decarboxylase activities in necropsy brain tissue. J Neurol Sci. 1977;34(2):247-265.

42. Danysz W, Parsons CG, Mobius HJ, Stoffler A, Quack G. Neuroprotective and symptomatological action of memantine relevant for Alzheimer's disease - a unified glutamatergic hypothesis on the mechanism of action. Neurotox Res. 2000;2(2-3):85-97.

43. Greenamyre JT, Maragos WF, Albin RL, Penney JB, Young AB. Glutamate transmission and toxicity in Alzheimer's disease. Prog Neuropsychopharmacol Biol Psychiatry. 1988;12(4):421-430.

44. Bilotta F, Doronzio A, Stazi E, et al. Postoperative cognitive dysfunction: toward the Alzheimer's disease pathomechanism hypothesis. J Alzheimers Dis. 2010;22(Suppl 3):81-89.

45. Eckenhoff RG, Johansson JS, Wei H, et al. Inhaled anesthetic enhancement of amyloid-beta oligomerization and cytotoxicity. Anesthesiology. 2004;101(3):703-709.

46. Dong Y, Zhang G, Zhang B, et al. The common inhalational anesthetic sevoflurane induces apoptosis and increases beta-amyloid protein levels. Arch Neurol. 2009;66(5):620-631.

47. Xie Z, Dong Y, Maeda U, et al. The common inhalation anesthetic isoflurane induces apoptosis and increases amyloid beta protein levels. Anesthesiology. 2006;104(5):988-994.

48. Perucho J, Rubio I, Casarejos MJ, et al. Anesthesia with isoflurane increases amyloid pathology in mice models of Alzheimer's disease. J Alzheimers Dis. 2010;19(4):1245-1257.

49. Whittington RA, Virág L, Marcouiller F, et al. Propofol directly increases tau phosphorylation. PLoS One. 2011;6(1):e16648.

50. Ma J, Shen B, Stewart LS, Herrick IA, Leung LS. The septohippocampal system participates in general anesthesia. J Neurosci. 2002; 22(2):RC200.

51. Anis NA, Berry SC, Burton NR, Lodge D. The dissociative anaesthetics, ketamine and phencyclidine, selectively reduce excitation of central mammalian neurones by N-methyl-aspartate. Br J Pharmacol. 1983;79(2):565-575.

52. Slikker W Jr, Zou X, Hotchkiss CE, et al. Ketamine-induced neuronal cell death in the perinatal rhesus monkey. Toxicol Sci. 2007;98(1):145-158.

53. Terrando N, Eriksson LI, Kyu Ryu J, et al. Resolving postoperative neuroinflammation and cognitive decline. Ann Neurol. 2011;70(6): 986-995.

54. Cibelli M, Fidalgo AR, Terrando N, et al. Role of interleukin-1beta in postoperative cognitive dysfunction. Ann Neurol. 2010;68(3):360-368.

55. Palotás A, Reis HJ, Bogáts G, et al. Coronary artery bypass surgery provokes Alzheimer's disease-like changes in the cerebrospinal fluid. J Alzheimers Dis. 2010;21(4):1153-1164.

56. Tang JX, Baranov D, Hammond M, Shaw LM, Eckenhoff MF, Eckenhoff RG. Human Alzheimer and inflammation biomarkers after anesthesia and surgery. Anesthesiology. 2011;115(4):727-732.

57. Shiiya N, Kunihara T, Miyatake T, Matsuzaki K, Yasuda K. Tau protein in the cerebrospinal fluid is a marker of brain injury after aortic surgery. Ann Thorac Surg. 2004;77(6):2034-2038.

58. Reinsfelt B, Ricksten SE, Zetterberg H, Blennow K, Fredén-Lindqvist J, Westerlind A. Cerebrospinal fluid markers of brain injury, inflammation, and blood-brain barrier dysfunction in cardiac surgery. Ann Thorac Surg. 2012;94(2):549-555.
59. Kimball CP. The experience of open heart surgery. 3. Toward a definition and understanding of postcardiotomy delirium. Arch Gen Psychiatry. 1972;27(1):57-63.

60. Berger M, Nadler J, Mathew JP. Preventing delirium after cardiothoracic surgery: provocative but preliminary evidence for bispectral index monitoring. Anesth Analg. 2014;118(4):706-707.

61. Radtke F, Franck M, Lendner J, Krüger S, Wernecke K, Spies C. Monitoring depth of anaesthesia in a randomized trial decreases the rate of postoperative delirium but not postoperative cognitive dysfunction. $\mathrm{Br}$ J Anaesth. 2013;110(Suppl 1):i98-i105.

62. Chan MT, Cheng BC, Lee TM, Gin T; CODA Trial Group. BIS-guided anesthesia decreases postoperative delirium and cognitive decline. J Neurosurg Anesthesiol. 2013;25(1):33-42.

63. Mason SE, Noel-Storr A, Ritchie CW. The impact of general and regional anesthesia on the incidence of post-operative cognitive dysfunction and post-operative delirium: a systematic review with metaanalysis. J Alzheimers Dis. 2010;22:67-79.

64. Slor CJ, de Jonghe JF, Vreeswijk R, et al. Anesthesia and postoperative delirium in older adults undergoing hip surgery. J Am Geriatr Soc. 2011;59(7):1313-1319.

65. Saczynski JS, Marcantonio ER, Quach L, et al. Cognitive trajectories after postoperative delirium. N Engl J Med. 2012;367(1):30-39.

66. Lundström M, Edlund A, Bucht G, Karlsson S, Gustafson Y. Dementia after delirium in patients with femoral neck fractures. $J$ Am Geriatr Soc. 2003;51(7):1002-1006.

67. Krogseth M, Wyller TB, Engedal K, Juliebø V. Delirium is an important predictor of incident dementia among elderly hip fracture patients. Dement Geriatr Cogn Disord. 2011;31(1):63-70.

68. Monk TG, Weldon BC, Garvan CW, et al. Predictors of cognitive dysfunction after major noncardiac surgery. Anesthesiology. 2008;108(1): 18-30.

69. Steinmetz J, Christensen KB, Lund T, Lohse N, Rasmussen LS; ISPOCD Group. Long-term consequences of postoperative cognitive dysfunction. Anesthesiology. 2009;110(3):548-555.

70. Avidan MS, Evers AS. Review of clinical evidence for persistent cognitive decline or incident dementia attributable to surgery or general anesthesia. J Alzheimers Dis. 2011;24(2):201-216.

71. Newman MF, Kirchner JL, Phillips-Bute B, et al; Neurological Outcome Research Group and the Cardiothoracic Anesthesiology Research Endeavors Investigators. Longitudinal assessment of neurocognitive function after coronary-artery bypass surgery. $N$ Engl J Med. 2001;344(6):395-402.

72. van Dijk D, Keizer A, Diephuis JC, Durand C, Vos LJ, Hijman R. Neurocognitive dysfunction after coronary artery bypass surgery: a systematic review. J Thorac Cardiovasc Surg. 2000;120(4):632-639.

73. Rörtgen D, Kloos J, Fries M, et al. Comparison of early cognitive function and recovery after desflurane or sevoflurane anaesthesia in the elderly: a double-blinded randomized controlled trial. Br J Anaesth. 2010;104(2):167-174.

74. Ballard C, Jones E, Gauge N, et al. Optimised anaesthesia to reduce post operative cognitive decline (POCD) in older patients undergoing elective surgery, a randomised controlled trial. PLoS One. 2012; 7(6):e37410.

75. Galasko D, Abramson I, Corey-Bloom J, Thal LJ. Repeated exposure to the Mini-Mental State Examination and the Information-MemoryConcentration Test results in a practice effect in Alzheimer's disease. Neurology. 1993;43(8):1559-1563.

76. Funder KS, Steinmetz J, Rasmussen LS. Methodological issues of postoperative cognitive dysfunction research. Semin Cardiothorac Vasc Anesth. 2010;14(2):119-122.

77. Abildstrom H, Rasmussen L, Rentowl P, et al. Cognitive dysfunction 1-2 years after non-cardiac surgery in the elderly. ISPOCD group. International Study of Post-Operative Cognitive Dysfunction. Acta Anaesthesiol Scand. 2000;44(10):1246-1251.

78. Canet J, Raeder J, Rasmussen L, et al; ISPOCD2 investigators. Cognitive dysfunction after minor surgery in the elderly. Acta Anaesthesiol Scand. 2003;47(10):1204-1210. 
79. Johnson T, Monk T, Rasmussen LS, et al; ISPOCD2 Investigators. Postoperative cognitive dysfunction in middle-aged patients. Anesthesiology. 2002;96(6):1351-1357.

80. Rasmussen L, Johnson T, Kuipers HM, et al; ISPOCD2 (International Study of Postoperative Cognitive Dysfunction) Investigators. Does anaesthesia cause postoperative cognitive dysfunction? A randomised study of regional versus general anaesthesia in 438 elderly patients. Acta Anaesthesiol Scand. 2003;47(3):260-266.

81. Steinmetz J, Siersma V, Kessing LV, Rasmussen LS; ISPOCD Group. Is postoperative cognitive dysfunction a risk factor for dementia? A cohort follow-up study. Br J Anaesth. 2013;110(Suppl 1):i92-i97.

82. Kudoh A, Ishihara H, Matsuki A. Response to surgical stress in elderly patients and Alzheimer's disease. Can J Anesth. 1999;46(3): 247-252.

83. van Harten AE, Scheeren TW, Absalom AR. A review of postoperative cognitive dysfunction and neuroinflammation associated with cardiac surgery and anaesthesia. Anaesthesia. 2012;67(3):280-293.

84. Chen PL, Yang CW, Tseng YK, et al. Risk of dementia after anaesthesia and surgery. Br J Psychiatry. 2014;204(3):188-193.

85. Lee TA, Wolozin B, Weiss KB, Bednar MM. Assessment of the emergence of Alzheimer's disease following coronary artery bypass graft surgery or percutaneous transluminal coronary angioplasty. J Alzheimers Dis. 2005;7(4):319-324.
86. Seitz DP, Shah PS, Herrmann N, Beyene J, Siddiqui N. Exposure to general anesthesia and risk of Alzheimer's disease: a systematic review and meta-analysis. BMC Geriatr. 2011;11(1):83.

87. Sprung J, Jankowski CJ, Roberts RO, et al. Anesthesia and incident dementia: a population-based, nested, case-control study. Mayo Clin Proc. 2013;88(6):552-561.

88. Chen CW, Lin CC, Chen KB, Kuo YC, Li CY, Chung CJ. Increased risk of dementia in people with previous exposure to general anesthesia: a nationwide population-based case-control study. Alzheimers Dement. 2014;10(2):196-204.

89. Vanderweyde T, Bednar MM, Forman SA, Wolozin B. Iatrogenic risk factors for Alzheimer's disease: surgery and anesthesia. J Alzheimers Dis. 2010;22(Suppl 3):91-104.

90. Liu Y, Pan N, Ma Y, et al. Inhaled sevoflurane may promote progression of amnestic mild cognitive impairment: a prospective, randomized parallel-group study. Am J Med Sci. 2013;345(5):355-360.
Clinical Interventions in Aging

\section{Publish your work in this journal}

Clinical Interventions in Aging is an international, peer-reviewed journal focusing on evidence-based reports on the value or lack thereof of treatments intended to prevent or delay the onset of maladaptive correlates of aging in human beings. This journal is indexed on PubMed Central, MedLine,

\section{Dovepress}

CAS, Scopus and the Elsevier Bibliographic databases. The manuscript management system is completely online and includes a very quick and fair peer-review system, which is all easy to use. Visit http://www.dovepress. com/testimonials.php to read real quotes from published authors. 